\title{
Manipulating topological spin textures in multiferroic and polar materials
}

\author{
J. Hugo Dil \\ Institut de Physique, Ecole Polytechnique Fédérale de Lausanne, \\ 1015 Lausanne, Switzerland \\ Photon Science Division, Paul Scherrer Institut, 5232 Villigen, Switzerland
}

\begin{abstract}
The possibility to manipulate spin textures is of central importance for their utilisation in functional materials. Here it will be shown how the resulting changes in the spin texture of the electronic structure can directly be imaged by spin- and angle-resolved photoemission spectroscopy (SARPES) by the examples of a multiferroic and a polar material. By using operando SARPES it will be shown that it is possible to reverse both the Rashbaand Zeeman-type spin textures in multiferroic $\mathrm{Ge}_{1-x} \mathrm{Mn}_{x}$ Te thin films by applying an external electric field in a capacitor type set-up. Furthermore, by growing homoepitaxial thin films of $\mathrm{SrTiO}_{3}(001)$ the filling of the 2D electron gas found in this system can be altered to achieve a single spin-polarised Fermi surface. Combined with the superconducting properties found in $\mathrm{SrTiO}_{3}$ this Fermi surface topology can form the starting point for the search of Majorana-type excitations.
\end{abstract}

Keywords: Spin textures, photoemission, operando measurements, ferroelectric, multiferroic, transition metal oxide, Majorana, 2DEG

\section{INTRODUCTION}

The classification of crystalline materials and their electronic structure by topological indices has gained much attention over the last decade. ${ }^{1-5}$ An important reason has been the mathematical and physical beauty of being able to summarise all possible band structures in a finite number of topological classes, which will each show a common response to excitations. However, from a more application focussed point of view, the interest in topology is mainly driven by the topologically protected, spin-polarised interface states that form at boundaries between regions with different bulk topology. This branch of topology primarily considers band crossings and inversions, and it should be noted that the occurrence of surface states in this case case is a direct consequence of the predictions by William Shockley in $1939 .{ }^{6}$ Their spin texture follows from the point symmetry at the surface or interface and for non-magnetic systems also has to obey time reversal symmetry. The simplest solutions is a single spin-polarised state with a helical spin texture as illustrated in Figure 1(a), representing the single Dirac cone surface state of a topological insulator (TI).

A similar situation can be achieved by starting with the two spin polarised bands formed under the influence of spin-orbit interaction (SOI) by the breaking of space inversion symmetry in a Rashba system ${ }^{7-9}$ and then gapping out one of the bands due to magnetic interactions, as illustrated in Figure 1(b). Although straightforward in theory, the practical application of the idea is more involved. An external magnetic field has to be large $(\gg 1 \mathrm{~T})$ in order to open a significant gap, which can cause further technical problems. Alternatively, the required magnetic interaction can be achieved by adding magnetic moments to the sample under investigation itself. In this case the magnetic moments should order along the same direction along which the symmetry is broken, and there should be a strong coupling to the bands with a Rashba-type spin splitting.

The difficulty in achieving these conditions is nicely illustrated by the study of a possible gap opening of the surface state of topological insulators. Both for magnetically doped topological insulators ${ }^{10,11}$ and layered intrinsically antiferromagnetic TIs ${ }^{12}$ a gap in the surface states due to magnetic order was claimed in photoemission

Further author information:

E-mail: hugo.dil@epfl.ch, Telephone: +41 (0)21 6935376 


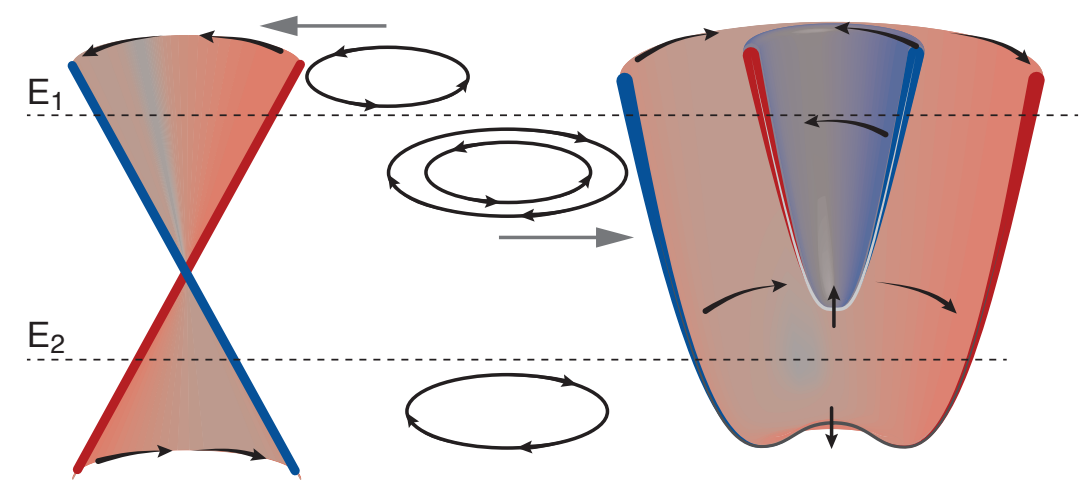

Figure 1. Dispersion and spin texture of (a) Dirac cone surface state of a topological insulator and (b) combined Rashba and Zeeman system. At energy $E_{1}$ the constant energy surfaces and spin texture for the two systems are distinct, whereas at $E_{2}$ inside the Zeeman gap they are identical.

experiments. However, separate measurements casted significant doubt on these claims, ${ }^{13-16}$ and also complimentary local probe measurements seem to indicate the absence of such a gap. ${ }^{17-19}$ For surface Rashba systems similar problems are encountered for out-of-plane magnetised systems, although for in-plane magnetisation the expected shift of the bands is observed. ${ }^{20,21}$ For bulk Rashba systems the coupling between the magnetic moments and the Rashba-split bulk bands appears to be stronger, and a Zeeman gap opening around the crossing point of the bands has been unambiguously observed both for Mn-doped GeTe ${ }^{22}$ and V-doped BiTeI. ${ }^{23}$

One possible explanation for the strong influence of the magnetism in the latter systems is that the origin of the magnetic order itself partly lies in the interaction of the magnetic moments with the spin-orbit split states of the valence band. Consequently, also the reverse influence is strong and a gap is opened up. In this light, the finding of a large Rashba-type spin splitting with a Zeeman gap in the two dimensional electron gas (2DEG) formed at the surface of $\mathrm{SrTiO}_{3}(001)^{24}$ (STO) is indicative of an intrinsic coupling between the origins of the magnetic and spin-orbit order. Also these are disputed, ${ }^{25}$ but differences are most likely due to sample preparation and surface quality. ${ }^{26}$ In any case, given the large possible impact of forming a single spin-polarised Fermi surface on an nominally insulating sample close to a superconducting transition, the system deserves further investigation and the second part of this paper is devoted to showing how the band filling can be changed to place the chemical potential in the Zeeman gap. ${ }^{26}$

\subsection{Spin- and Angle Resolved Photoemission Spectroscopy}

With the exception of skyrmions and related systems where the influence of SOI and exchange interaction are well balanced in the Dzyaloshinskii Moriya interaction, the spin textures related to strong SOI are only detectable in reciprocal space techniques. With the possibility to measure the spin polarisation vector for every point of the occupied band structure in reciprocal space, spin- and angle-resolved photoemission spectroscopy (SARPES) is thus the method of choice. The details, possibilities, and challenges of SARPES have been reviewed elsewhere, ${ }^{27,28}$ including recent technical developments, ${ }^{29}$ and a special focus on topological materials. ${ }^{30}$

In short, similar to typical angle-resolved photoemission (ARPES) the band structure is determined by measuring the kinetic energy and emission angle of electrons emitted by a monochromatic light source and transforming the obtained values to binding energy and momentum. However, instead of directly detecting the electron, it is passed on to a spin detector where the spin expectation value is determined by a scattering technique, thereby yielding a 3D spin vector for each band under investigation. Without going into detail, two points should be noted. First, excitation with circularly polarised light does not yield information about the spin but about the orbital angular momentum, which can be parallel or anti-parallel to the spin. Second, SARPES does not necessarily detect the initial state spin texture, but rather its convolution with the photoemission matrix elements. For further details the reader is referred to the articles mentioned above. 


\section{SPIN TEXTURE MANIPULATION IN MULTIFERROIC $\mathrm{Ge}_{1-x} \mathrm{Mn}_{x} \mathrm{Te}$}

In order to utilise the topological spin textures found in systems with strong SOI it is of central importance to be able to manipulate them. The most extreme case is the reversal of the spin texture in ferroelectric materials upon switching the ferroelectric polarisation direction. ${ }^{31}$ The use of ferroelectric materials has the additional advantage that the changes can be stored in a non-volatile manner. The experimental verification that the reversal of the crystal polarisation does indeed lead to a reversal of the Rashba-type spin texture is thus of importance to open up a further field of study and applications.

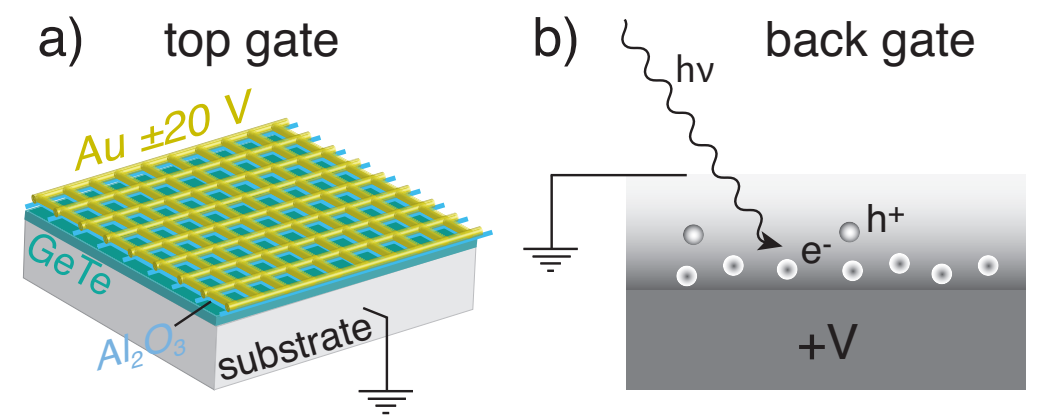

Figure 2. (a) Schematic of sample mounting used for operando SARPES experiments on GeTe(111) films. The voltage is applied to the Au mesh on the top to ensure that the sample surface is sensitive to it. (b) Schematic for a back gating experiment. In this case the free charges created by the photon beam will rapidly screen the back gate voltage and at the sample surface no additional potential gradient is present and no operando effects can be expected.

Although straightforward in theory, the experimental realisation of SARPES measurements on ferroelectrically switched crystals is much more involved. The most important reason is that in order to change the polarisation one has to place the sample between two electrodes, whereby the top electrode would directly block the incoming light and outgoing photoelectrons. An elegant solution to this problem would be to use graphene as a top gate recently done for normal ARPES. ${ }^{32}$ However, this limits the possibility of in situ surface preparation and is currently primarily feasible in combination with other non-reactive van der Waals materials. Thus, until a method to transfer and contact graphene under UHV conditions is found, another method has to be used for most materials. We decided to use a gold mesh with a large open area and chose the dimensions such as to have a homogeneous electric field in the holes of the mesh at the sample surface. To ensure electrical isolation between the sample and mesh, the latter was coated by a thin dielectric layer. This way the sample surface could be prepared by our standard decapping method of thin GeTe(111) films. ${ }^{33}$ Figure 2(a) shows a schematic of the sample mounting and further details can be found in the original publication. ${ }^{34}$

Another problem of performing operando (S)ARPES experiments is that the large amount of free charges that are created by the photon beam (e.g. secondary electrons) will screen the applied voltages. It is therefore crucial to apply the voltage as close as possible to the region one is interested in. In most (S)ARPES experiments this is the sample surface, which renders so-called back gating useless as illustrated in Figure 2(b) unless few monolayer thin samples are investigated. In our operando SARPES experiments we therefore applied a voltage to the mesh while keeping the metallic substrate of the the $200 \mathrm{~nm} \mathrm{Ge} \mathrm{Ge}_{1-x} \mathrm{Mn}_{x} \mathrm{Te}(111)$ film at ground potential. As a result, the kinetic energy of the bands and Fermi level is shifted, but this is a small price to pay and can easily be corrected.

\subsection{Spin Texture Inversion in Ferroelectric GeTe}

Our main finding for operando SARPES experiments on ferroelectric GeTe(111) films are shown in Figure 3. As schematically illustrated in Figure 3(a), the Rashba-type spin texture is expected to be inverted upon reversal of the ferroelectric polarisation of the thin films. The spin polarisation spectra as function of momentum in Figure $3(\mathrm{~b})$ show exactly this type of reversal upon applying a $-1.6 \mathrm{~V}$ to the Au mesh; the $x, y$, and $z$ component of the spin polarisation are all reversed, although their magnitude is reduced. This reduction can be partly attributed to the lower data quality when measuring with an applied voltage, but it is also an indication that not the entire 
probed region of $800 \times 200 \mu \mathrm{m}$ is switched. This limited switching is probably related to the pinning of domains at defects and domain walls and becomes even clearer when subsequently a $+1.6 \mathrm{~V}$ bias is applied to the mesh. Now $P_{x}$ and $P_{z}$ switch with strongly reduced magnitude, but $P_{y}$ does not invert at all and the magnitude is even increased.

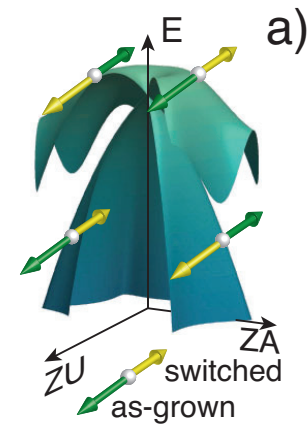

b)

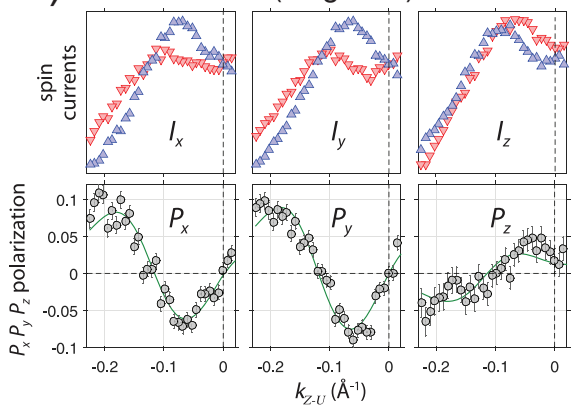

c)
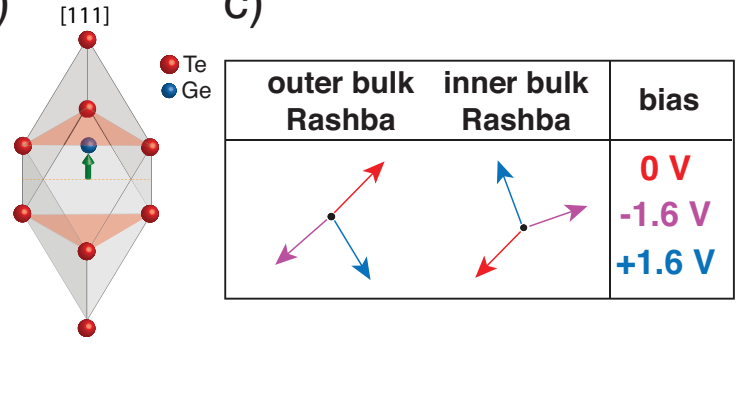

d)

\begin{tabular}{|c|c|c|c|c|}
\hline \multirow{2}{*}{ 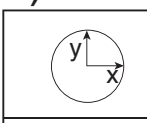 } & OS OB & IS & IB & \multirow{2}{*}{$\begin{array}{c}\text { Gate } \\
\text { voltage }\end{array}$} \\
\hline & \multicolumn{3}{|c|}{$\cdots$, spinors before annealing } & \\
\hline $\begin{array}{c}\text { first } \\
\text { reannealing }\end{array}$ & 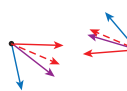 & & & $\begin{array}{r}0 \mathrm{~V} \\
+10 \mathrm{~V} \\
-10 \mathrm{~V}\end{array}$ \\
\hline $\begin{array}{l}\text { second } \\
\text { reannealing }\end{array}$ & 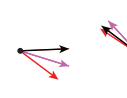 & & $\hat{\lambda}$ & $\begin{array}{c}0 \mathrm{~V} \\
-10 \mathrm{~V} \\
-17 \mathrm{~V}\end{array}$ \\
\hline
\end{tabular}
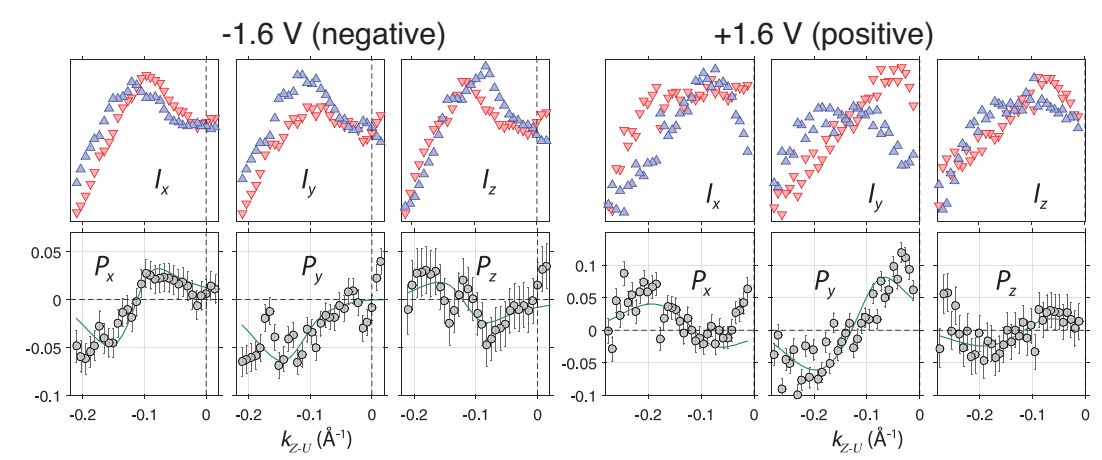

Figure 3. (a) Schematic of the band structure and spin texture of the valence band (left) and crystal structure (right) of GeTe(111). The spin orientation reverses with a reversal of the ferroelectric displacement. (b) Spin resolved momentum distribution curves of the Rashba split bulk bands for different voltages applied to the Au mesh. (c) Summary of the obtained in-plane spin vectors for the different measurements in (b). (d) Spin vectors as a function of applied voltage and annealing step for SARPES data obtained on a slightly optimised sample design and as a function of energy. O(I)S is the outer (inner) surface resonance and $\mathrm{O}(\mathrm{I}) \mathrm{B}$ the outer (inner) bulk band. Figure adapted from 34.

This effect is known as ferroelectric fatigue and is a well known problem in many ferroelectric materials. ${ }^{35}$ An efficient way to verify whether it is truly fatigue which is limiting the switching is to anneal the sample, but well below the ferroelectric transition temperature. This way trapped depolarising charges are released and a "pristine" polarised state is restored. The results of such annealing steps are shown in Figure 3(d) and after each anneal it is possible to switch the spin texture once, after which it is stalled. These data were obtained scanning the binding energy for an optimised sample holder design which allowed for multiple temperature cycles. Under this measurement geometry a hybridisation between surface (OS) and bulk (OB) derived states is influencing the data interpretation, the details of which go beyond the scope of this report and are discussed in the original publication. ${ }^{34}$ For possible applications it is interesting to note that, even though spin is no longer a good quantum number under the influence of SOI, around the valance band maximum the bulk states of GeTe are fully spin polarised also in the initial state. ${ }^{36}$

\subsection{Changing Magnetism by Electric Field in Multiferroic $\mathbf{G e}_{1-x} \mathbf{M n}_{x} \mathbf{T e}$}

The opening of a Zeeman gap in a Rashba system should lead to an out-of-plane spin polarisation around the gap edges. This is exactly what is observed for $\mathrm{Ge}_{1-x} \mathrm{Mn}_{x} \mathrm{Te}(111)$ thin films, whereby this $P_{z}$ can be remanently switched by a small external magnetic field as shown in Figure 4(a). ${ }^{22}$ As explained above, the opening of such a gap is indicative of a strong interaction between the spin-orbit split bands and the local magnetic moments, which poses the question whether the magnetic orientation can also be altered by changing the spin texture of 
the Rashba-split bands by an electric field as shown in the previous section. This would be indicative of a strong magnetoelectric coupling and show that it is possible to switch magnetic domains purely by electric fields.

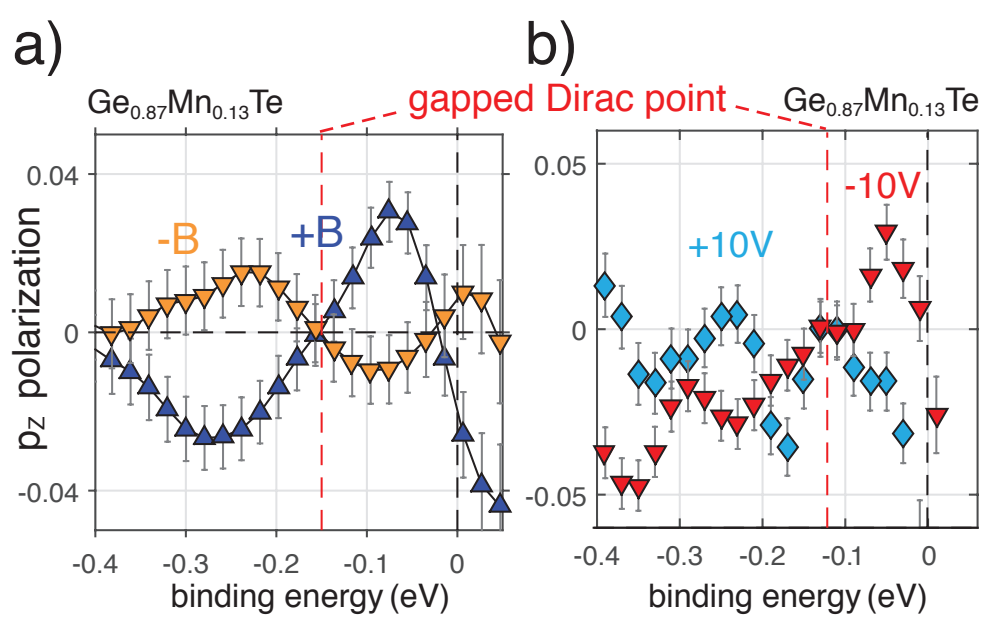

Figure 4. Switching of the magnetic spin texture around the Zeeman gap in $\mathrm{Ge}_{0.87} \mathrm{Mn}_{0.13} \mathrm{Te}(111)$ films by an external magnetic field of \pm 700 Gauss (a) or electric field of $\pm 10 \mathrm{~V}$ (b). Figure adapted from 22,34.

The results of the operando SARPES experiments where an electric field is applied to a Au mesh placed on top of $200 \mathrm{~nm}$ thick $\mathrm{Ge}_{0.87} \mathrm{Mn}_{0.13} \mathrm{Te}(111)$ films are summarised in Figure 4(b). Although a switching is achieved, it is not perfect and the pathway appears more complex as just a simple back and forth switch. ${ }^{34}$ Whether this is related to the ferroelectric fatigue discussed above, or whether this is due to the intrinsic physical nature of the coupling mechanism, requires further investigation. On the one hand it would be good to repeat the measurements with higher quality samples showing less fatigue, but more detailed information can especially be obtained from measurements combining electric and magnetic fields. This goes currently beyond the capabilities of existing SARPES set-ups and requires further technical developments.

Before continuing with the next topic of a single spin-polarised Fermi surface in $\mathrm{SrTiO}_{3}$ thin films we would like to stress that the operando SARPES experiments described above were the first measurements of their kind. The main aim is to show that such experiments are possible and directly provide a large amount of information. However, to make a direct link with functional materials further improvements in sample quality and type, and especially in the way the voltage is applied and the general SARPES set-up itself, are required.

\section{SINGLE SPIN-POLARISED FERMI SURFACE ON $\mathrm{SrTiO}_{3} \mathrm{HOMOEPITAXIAL}$ FILMS}

One of the reasons why topological insulators stirred much enthusiasm in the community is the prospect of having a single spin-polarised Fermi surface on a further insulating bulk system. The electromagnetic response of this Fermi surface would be similar to a magnetic monopole ${ }^{37}$ and in combination with superconductivity it would form the basis for creating quasiparticles resembling Majorana fermions. ${ }^{38}$ Because the response of topological insulators is the same as for narrow band gap semiconductors with regard to surface band bending, one of the problems is to find a topological insulator where the bulk bands don't cross the Fermi level in either the bulk or near surface region. ${ }^{39}$ Using careful element substitution that problem could be resolved and exciting experiments requiring insulating character throughout the sample are now possible. ${ }^{40}$ However, interfacing with a superconductor can rapidly tip this delicate balance and up to now no convincing reports on inducing superconductivity in a bulk insulating TI exist to our best knowledge. Therefore, other methods to form a single spin-polarised Fermi surface in materials that are easier to handle is required and the combined Rashba and Zeeman spin splitting described above is the most promising recipe.

Figure 5(a) summarises the band structure and the spin texture obtained for the 2DEG found at the surface of $\mathrm{SrTiO}_{3}(001)$ wafers. ${ }^{24,41,42}$ The $d_{x y}$-derived bands show the required Rashba-type spin texture in combination 
with a gap at the zone centre, but this is significantly below the Fermi level and at the Fermi level there are additional (spin-degenerate) $d_{x z}$ and $d_{y z}$ derived bands. In order to alter the electronic structure and place the chemical potential in the Zeeman gap we grew thin STO films on Nb-doped STO(001) substrates by pulsed laser deposition, the details of which are described elsewhere. ${ }^{26}$ The findings for these films are independent on thickness in the studied range between 3 and 20 unit cells (u.c.), indicating that confinement effects play no important role and also that the $2 \mathrm{DEG}$ extends 2 u.c. or less from the surface. On the other hand, the properties do depend on the $\mathrm{Nb}$ doping of the substrate and especially on the amount of $\mathrm{SrO}_{x}$ at the sample surface. ${ }^{26}$
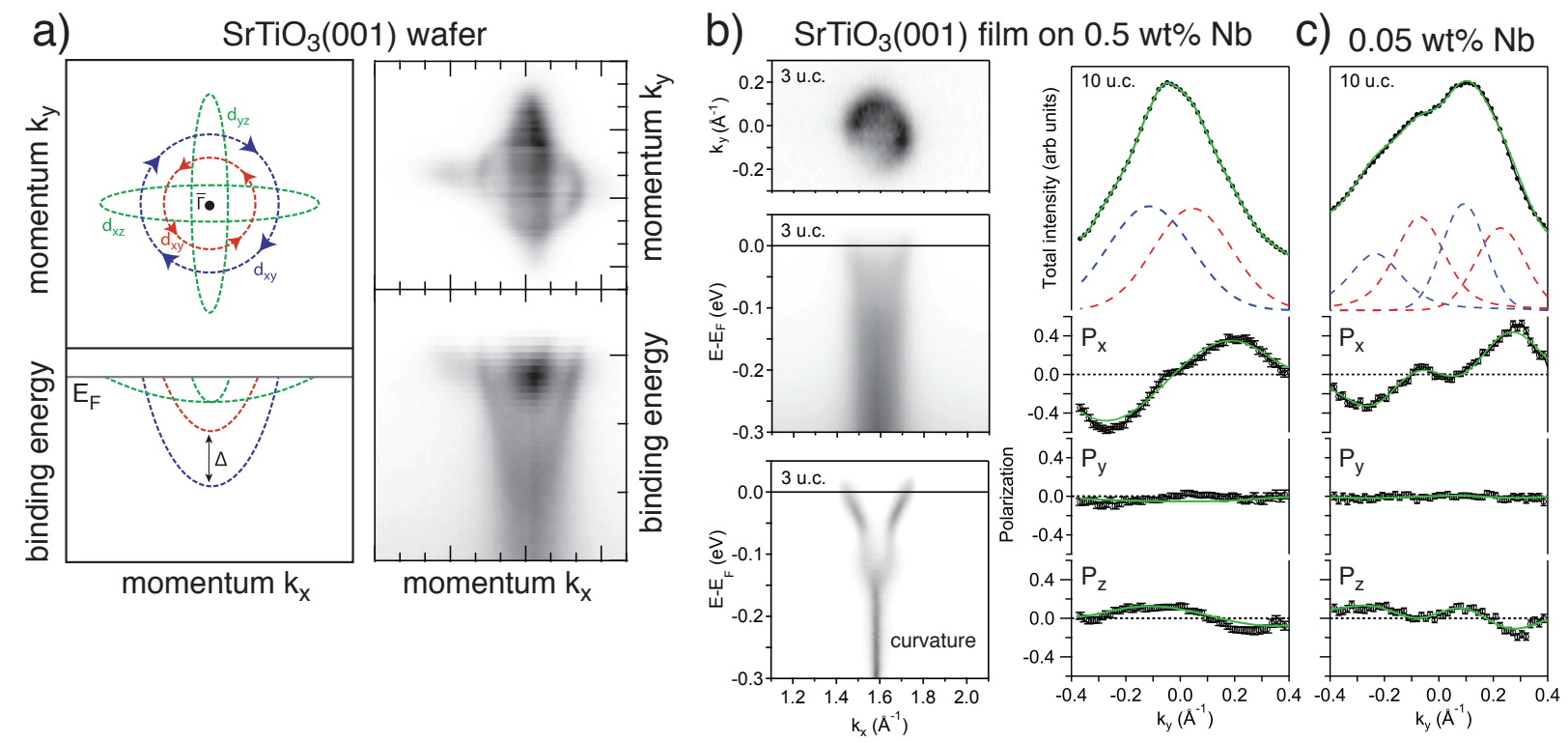

Figure 5. (a) Schematic and measured band structure of the $2 \mathrm{DEG}$ on $\mathrm{SrTiO}_{3}(001)$ wafers with the spin texture overlaid on the sketch. (b) Measured band structure and curvature fit of a 3 u.c. thick $\mathrm{SrTiO}_{3}$ film and spin resolved momentum distribution curve with all three polarisation components obtained at the Fermi level for the 2DEG on a 10 u.c. thick film. Both films are grown on a $0.5 \mathrm{wt} \% \mathrm{Nb}$ doped $\mathrm{STO}(001)$ substrate. (c) Similar spin-resolved data obtained for a 10 u.c. thick film grown on a $0.05 \mathrm{wt} \% \mathrm{Nb}$ doped $\mathrm{STO}(001)$ substrate. Figure adapted from 26.

In Figure 5(b) the measured electronic structure and spin texture of a 3 u.c. and 10 u.c. thick film respectively, both grown on $0.5 \mathrm{wt} \% \mathrm{Nb}$-doped STO substrates, are displayed. From the comparison with Figure 5(a) it is directly clear that the $d_{x z}$ and $d_{y z}$ derived bands are not occupied and that the 2DEG has been shifted towards the Fermi level by about $160 \mathrm{meV}$. As a result there appears to be only a single parabolic band crossing the Fermi level. From the SARPES data it follows that this is indeed a single spin-polarised state with a helical spin texture, reminiscent of what is observed for the surface state of topological insulators. ${ }^{30}$

Interestingly, the band filling of the 2DEG increases when similar films are grown on $0.05 \mathrm{wt} \% \mathrm{Nb}$-doped STO substrates, and as shown in Figure 5(c) in this case two bands with opposite spin texture cross the Fermi level. ${ }^{26}$ Whether this change is due to an altered dielectric response of the film to the fact that the substrate doping is changed by an order of magnitude, or that it is due to changes in the film stoichiometry or structure, requires further study. It is certain that the change is not due to charge transfer from the substrate because the band shifts in the opposite direction. Furthermore, it remains to be verified whether the thin STO films become superconducting at low temperatures. The substrates, however, are expected to be superconducting below $300 \mathrm{mK}^{43}$ and could induce superconducting properties in the films by proximity effect.

The single spin-polarised Fermi surface without other bands present, and the induced superconductivity unite the required properties for the formation of Majorana bound states. ${ }^{38,44}$ Starting from the 2D platform structures at whose tips Majorana bounds states should form can be written by substrate lithography, EUV photons, or a conductive tip. ${ }^{45}$ Depending on the shape of the structures these can be used for braiding or other manipulation of these still rather elusive quasiparticles as required for quantum computing applications. 


\section{ACKNOWLEDGMENTS}

This work was supported by the Swiss National Science Foundation (SNF) Project No. PP00P2_144742 and No. PP00P2_170591. The author thanks Juraj Krempasky, Stefan Muff, Eduardo Guedes, and Milan Radovic for their crucial contributions to the research discussed in this report.

\section{REFERENCES}

[1] Hasan, M. Z. and Kane, C. L., "Colloquium: Topological insulators," Reviews of Modern Physics 82 (11 2010).

[2] Qi, X.-L. and Zhang, S.-C., "Topological insulators and superconductors," Reviews of Modern Physics 83, 1057-1110 (10 2011).

[3] Haldane, F. D. M., "Nobel lecture: Topological quantum matter," Reviews of Modern Physics 89, 040502(10 2017).

[4] Kosterlitz, J. M., "Nobel lecture: Topological defects and phase transitions," Reviews of Modern Physics 89, 040501- (10 2017).

[5] Armitage, N. P., Mele, E. J., and Vishwanath, A., "Weyl and dirac semimetals in three-dimensional solids," Reviews of Modern Physics 90, 015001- (01 2018).

[6] Shockley, W., "On the surface states associated with a periodic potential," Physical Review 56, 317-323 (08 1939).

[7] Bychkov, Y. A. and Rashba, E. I., "Oscillatory effects and the magnetic susceptibility of carriers in inversion layers," Journal of Physics C: Solid State Physics 17(33), 6039-6045 (1984).

[8] LaShell, S., McDougall, B. A., and Jensen, E., "Spin splitting of an Au(111) surface state band observed with angle resolved photoelectron spectroscopy," Phys. Rev. Lett. 77, 3419-3422 (Oct 1996).

[9] Ast, C. R., Henk, J., Ernst, A., Moreschini, L., Falub, M. C., Pacilé, D., Bruno, P., Kern, K., and Grioni, M., "Spin splitting through surface alloying," Physical Review Letters 98(18), 186807 (2007).

[10] Chen, Y. L., Chu, J. H., Analytis, J. G., Liu, Z. K., Igarashi, K., Kuo, H. H., Qi, X. L., Mo, S. K., Moore, R. G., Lu, D. H., Hashimoto, M., Sasagawa, T., Zhang, S. C., Fisher, I. R., Hussain, Z., and Shen, Z. X., "Massive dirac fermion on the surface of a magnetically doped topological insulator," Science 329, 659-662 (08 2010).

[11] Xu, S.-Y., Neupane, M., Liu, C., Zhang, D., Richardella, A., Andrew Wray, L., Alidoust, N., Leandersson, M., Balasubramanian, T., Sanchez-Barriga, J., Rader, O., Landolt, G., Slomski, B., Dil, J. H., Osterwalder, J., Chang, T.-R., Jeng, H.-T., Lin, H., Bansil, A., Samarth, N., and Zahid Hasan, M., "Hedgehog spin texture and berry's phase tuning in a magnetic topological insulator," Nature Physics 8, 616-622 (08 2012).

[12] Rienks, E. D. L., Wimmer, S., Sánchez-Barriga, J., Caha, O., Mandal, P. S., Růžička, J., Ney, A., Steiner, H., Volobuev, V. V., Groiss, H., Albu, M., Kothleitner, G., Michalička, J., Khan, S. A., Minár, J., Ebert, H., Bauer, G., Freyse, F., Varykhalov, A., Rader, O., and Springholz, G., "Large magnetic gap at the dirac point in $\mathrm{Bi}_{2} \mathrm{Te}_{3} / \mathrm{MnBi}_{2} \mathrm{Te}_{4}$ heterostructures," Nature 576(7787), 423-428 (2019).

[13] Sánchez-Barriga, J., Varykhalov, A., Springholz, G., Steiner, H., Kirchschlager, R., Bauer, G., Caha, O., Schierle, E., Weschke, E., Ünal, A. A., Valencia, S., Dunst, M., Braun, J., Ebert, H., Minár, J., Golias, E., Yashina, L. V., Ney, A., Holý, V., and Rader, O., "Nonmagnetic band gap at the dirac point of the magnetic topological insulator $\left(\mathrm{Bi}_{1 x} \mathrm{Mn}_{x}\right)_{2} \mathrm{Se}_{3}, "$ Nature Communications 7, 10559 (02 2016).

[14] Li, H., Gao, S.-Y., Duan, S.-F., Xu, Y.-F., Zhu, K.-J., Tian, S.-J., Gao, J.-C., Fan, W.-H., Rao, Z.-C., Huang, J.-R., Li, J.-J., Yan, D.-Y., Liu, Z.-T., Liu, W.-L., Huang, Y.-B., Li, Y.-L., Liu, Y., Zhang, G.-B., Zhang, P., Kondo, T., Shin, S., Lei, H.-C., Shi, Y.-G., Zhang, W.-T., Weng, H.-M., Qian, T., and Ding, H., "Dirac surface states in intrinsic magnetic topological insulators $\mathrm{EuSn}_{2} \mathrm{As}_{2}$ and $\mathrm{MnBi}_{2 n} \mathrm{Te}_{3 n+1}$," Physical Review X 9, 041039- (11 2019).

[15] Hao, Y.-J., Liu, P., Feng, Y., Ma, X.-M., Schwier, E. F., Arita, M., Kumar, S., Hu, C., Lu, R., Zeng, M., Wang, Y., Hao, Z., Sun, H.-Y., Zhang, K., Mei, J., Ni, N., Wu, L., Shimada, K., Chen, C., Liu, Q., and Liu, C., "Gapless surface dirac cone in antiferromagnetic topological insulator $\mathrm{MnBi}_{2} \mathrm{Te}_{4}$," Physical Review $X$ 9, 041038-(11 2019). 
[16] Chen, Y. J., Xu, L. X., Li, J. H., Li, Y. W., Wang, H. Y., Zhang, C. F., Li, H., Wu, Y., Liang, A. J., Chen, C., Jung, S. W., Cacho, C., Mao, Y. H., Liu, S., Wang, M. X., Guo, Y. F., Xu, Y., Liu, Z. K., Yang, L. X., and Chen, Y. L., "Topological electronic structure and its temperature evolution in antiferromagnetic topological insulator $\mathrm{MnBi}_{2} \mathrm{Te}_{4}, "$ Physical Review X 9, 041040- (11 2019).

[17] Beidenkopf, H., Roushan, P., Seo, J., Gorman, L., Drozdov, I., Hor, Y. S., Cava, R. J., and Yazdani, A., "Spatial fluctuations of helical dirac fermions on the surface of topological insulators," Nature Physics $\mathbf{7}(12)$, 939-943 (2011).

[18] Yuan, Y., Wang, X., Li, H., Li, J., Ji, Y., Hao, Z., Wu, Y., He, K., Wang, Y., Xu, Y., Duan, W., Li, W., and Xue, Q.-K., "Electronic states and magnetic response of $\mathrm{MnBi}_{2} \mathrm{Te}_{4}$ by scanning tunneling microscopy and spectroscopy," Nano Letters 20, 3271-3277 (05 2020).

[19] Wu, X., Li, J., Ma, X.-M., Zhang, Y., Liu, Y., Zhou, C.-S., Shao, J., Wang, Q., Hao, Y.-J., Feng, Y., Schwier, E. F., Kumar, S., Sun, H., Liu, P., Shimada, K., Miyamoto, K., Okuda, T., Wang, K., Xie, M., Chen, C., Liu, Q., Liu, C., and Zhao, Y., "Distinct topological surface states on the two terminations of $\mathrm{MnBi}_{4} \mathrm{Te}_{7}, "$ Physical Review X 10, 031013- (07 2020).

[20] Krupin, O., Bihlmayer, G., Starke, K., Gorovikov, S., Prieto, J. E., Dobrich, K., Blugel, S., and Kaindl, G., "Rashba effect at magnetic metal surfaces," Physical Review B 71, 201403 (May 2005).

[21] Usachov, D. Y., Güttler, M., Schulz, S., Poelchen, G., Seiro, S., Kliemt, K., Kummer, K., Krellner, C., Laubschat, C., Chulkov, E. V., and Vyalikh, D. V., "Spin structure of spin-orbit split surface states in a magnetic material revealed by spin-integrated photoemission," Physical Review B 101, 245140- (06 2020).

[22] Krempaský, J., Muff, S., Bisti, F., Fanciulli, M., Volfová, H., Weber, A. P., Pilet, N., Warnicke, P., Ebert, H., Braun, J., Bertran, F., Volobuev, V. V., Minár, J., Springholz, G., Dil, J. H., and Strocov, V. N., "Entanglement and manipulation of the magnetic and spin-orbit order in multiferroic rashba semiconductors," Nature Communications 7, 13071 (10 2016).

[23] Shikin, A. M., Rybkina, A. A., Klimovskikh, I. I., Tereshchenko, O. E., Bogomyakov, A. S., Kokh, K. A., Kimura, A., Skirdkov, P. N., Zvezdin, K. A., and Zvezdin, A. K., "Anomalously large gap and induced outof-plane spin polarization in magnetically doped 2D rashba system: V-doped BiTeI," 2D Materials 4(2), 025055 (2017).

[24] Santander-Syro, A. F., Fortuna, F., Bareille, C., Rödel, T. C., Landolt, G., Plumb, N. C., Dil, J. H., and Radović, M., "Giant spin splitting of the two-dimensional electron gas at the surface of $\mathrm{SrTiO}_{3}$," Nature materials 13(12), 1085-1090 (2014).

[25] McKeown Walker, S., Riccò, S., Bruno, F. Y., de la Torre, A., Tamai, A., Golias, E., Varykhalov, A., Marchenko, D., Hoesch, M., Bahramy, M. S., King, P. D. C., Sánchez-Barriga, J., and Baumberger, F., "Absence of giant spin splitting in the two-dimensional electron liquid at the surface of $\mathrm{SrTiO}_{3}(001)$," Physical Review B 93, 245143 (06 2016).

[26] Guedes, E. B., Muff, S., Fanciulli, M., Weber, A. P., Caputo, M., Wang, Z., Plumb, N. C., Radović, M., and Dil, J. H., "Single spin-polarized fermi surface in $\mathrm{SrTiO}_{3}$ thin films," Physical Review Research 2, 033173(07 2020).

[27] Dil, J. H., "Spin and angle resolved photoemission on non-magnetic low-dimensional systems," Journal of Physics: Condensed Matter 21(40), 403001 (2009).

[28] Heinzmann, U. and Dil, J. H., "Spin-orbit-induced photoelectron spin polarization in angle-resolved photoemission from both atomic and condensed matter targets," Journal of Physics: Condensed Matter 24(17), 173001 (2012).

[29] Okuda, T., "Recent trends in spin-resolved photoelectron spectroscopy," Journal of Physics: Condensed Matter 29(48), 483001 (2017).

[30] Dil, J. H., "Spin- and angle-resolved photoemission on topological materials," Electronic Structure 1(2), 023001 (2019).

[31] Di Sante, D., Barone, P., Bertacco, R., and Picozzi, S., "Electric Control of the Giant Rashba Effect in Bulk GeTe," Advanced Materials 25(4), 509-513 (2013).

[32] Nguyen, P. V., Teutsch, N. C., Wilson, N. P., Kahn, J., Xia, X., Graham, A. J., Kandyba, V., Giampietri, A., Barinov, A., Constantinescu, G. C., Yeung, N., Hine, N. D. M., Xu, X., Cobden, D. H., and Wilson, N. R., "Visualizing electrostatic gating effects in two-dimensional heterostructures," Nature 572(7768), 220-223 (2019). 
[33] Krempaský, J., Volfová, H., Muff, S., Pilet, N., Landolt, G., Radović, M., Shi, M., Kriegner, D., Holý, V., Braun, J., Ebert, H., Bisti, F., Rogalev, V. A., Strocov, V. N., Springholz, G., Minár, J., and Dil, J. H., "Disentangling bulk and surface Rashba effects in ferroelectric $\alpha$-GeTe," Phys. Rev. B 94, 205111 (Nov 2016).

[34] Krempaský, J., Muff, S., Minár, J., Pilet, N., Fanciulli, M., Weber, A. P., Guedes, E. B., Caputo, M., Müller, E., Volobuev, V. V., Gmitra, M., Vaz, C. A. F., Scagnoli, V., Springholz, G., and Dil, J. H., "Operando imaging of all-electric spin texture manipulation in ferroelectric and multiferroic rashba semiconductors," Physical Review X 8, 021067- (06 2018).

[35] Lupascu, D. C., ed., [Fatigue in Ferroelectric Ceramics and Related Issues], ser. Physics and Astronomy Online Library. Springer, Berlin (2004).

[36] Krempaský, J., Fanciulli, M., Nicolaï, L., Minár, J., Volfová, H., Caha, O., Volobuev, V. V., SánchezBarriga, J., Gmitra, M., Yaji, K., Kuroda, K., Shin, S., Komori, F., Springholz, G., and Dil, J. H., "Fully spin-polarized bulk states in ferroelectric GeTe," Physical Review Research 2, 013107- (01 2020).

[37] Qi, X.-L., Li, R., Zang, J., and Zhang, S.-C., "Inducing a magnetic monopole with topological surface states," Science 323, 1184 (02 2009).

[38] Beenakker, C. W. J., "Search for majorana fermions in superconductors," Annual Review of Condensed Matter Physics 4, 113-136 (2020/07/24 2013).

[39] Muff, S., von Rohr, F., Landolt, G., Slomski, B., Schilling, A., Cava, R. J., Osterwalder, J., and Dil, J. H., "Separating the bulk and surface n- to p-type transition in the topological insulator $\mathrm{GeBi}_{4-x} \mathrm{Sb}_{x} \mathrm{Te}_{7}$," Physical Review B 88, 035407- (07 2013).

[40] Reimann, J., Schlauderer, S., Schmid, C. P., Langer, F., Baierl, S., Kokh, K. A., Tereshchenko, O. E., Kimura, A., Lange, C., Güdde, J., Höfer, U., and Huber, R., "Subcycle observation of lightwave-driven dirac currents in a topological surface band," Nature 562(7727), 396-400 (2018).

[41] Santander-Syro, A. F., Copie, O., Kondo, T., Fortuna, F., Pailhès, S., Weht, R., Qiu, X. G., Bertran, F., Nicolaou, A., Taleb-Ibrahimi, A., Fèvre, P. L., Herranz, G., Bibes, M., Reyren, N., Apertet, Y., Lecoeur, P., Barthélémy, A., and Rozenberg, M. J., "Two-dimensional electron gas with universal subbands at the surface of $\mathrm{SrTiO}_{3}, "$ Nature 469, 189 (2011).

[42] Plumb, N. C., Salluzzo, M., Razzoli, E., Månsson, M., Falub, M., Krempasky, J., Matt, C. E., Chang, J., Schulte, M., Braun, J., Ebert, H., Minár, J., Delley, B., Zhou, K.-J., Schmitt, T., Shi, M., Mesot, J., Patthey, L., and Radović, M., "Mixed dimensionality of confined conducting electrons in the surface region of $\mathrm{SrTiO}_{3}$," Phys. Rev. Lett. 113, 086801 (Aug 2014).

[43] Schooley, J. F., Hosler, W. R., and Cohen, M. L., "Superconductivity in semiconducting $\mathrm{SrTiO}_{3}$," Phys. Rev. Lett. 12, 474-475 (Apr 1964).

[44] Mourik, V., Zuo, K., Frolov, S. M., Plissard, S. R., Bakkers, E. P. A. M., and Kouwenhoven, L. P., "Signatures of majorana fermions in hybrid superconductor-semiconductor nanowire devices," Science 336, 1003-1007 (05 2012).

[45] Cen, C., Thiel, S., Hammerl, G., Schneider, C., Andersen, K., Hellberg, C., Mannhart, J., and Levy, J., "Nanoscale control of an interfacial metal-insulator transition at room temperature," Nature materials 7(4), 298-302 (2008). 